\title{
Near-Best Approximation by a de la Vallée Poussin-type Interpolatory Operator
}

\author{
Ágota P. Horváth * \\ Department of Analysis, Budapest University of Technology and Economics \\ H-1521 Budapest, Hungary \\ e-mail: ahorvath@renyi.hu
}

\begin{abstract}
We give a very simply computable interpolatory process, wich approximates in near-best order on $[-1,1]$ in some Jacobi-weighted space.
\end{abstract}

\section{Introduction, Definitions}

Practically, it is always an interesting problem to construct a discrete linear operator, which approximates for instance continuous functions in near-best order. It is a natural idea to use some kind of de la Vallée Poussin-type means for proving best, or near-best order of approximation. After the investigations of eg. R. Bojanic, O. Shisha [1], and G. Freud [2], in 1974 J. Szabados gave the discrete version of de la Vallée Poussin means in the trigonometric case [11]. The considerable generalization of this result, which was simultaneously an answere of a question of G. Freud and A. Sharma [3], was given by O. Kis and J. Szabados [4].

In 1999 H. N. Mhaskar and J. Prestin established a result on bounded quasiinterpolatory operators [9], which also based on de la Vallée Poussin-type means. Their discretizing method based on the quadrature formula, and not on some integral approximating sum, as in the previous papers. However it deals with rather general weighted spaces, (applying the results to generalized Jacobi and Freud weights) the interpolatory property had been lost. In this direction some more investigations appeared eg: [5], [7], [8]. Generalized de la Vallée Poussin means in Jacobi-weighted $L^{p}$-spaces was first treated by Nguyen Xuan Ky [10], and in 2008 further results were given by G. Mastroianni and W. Themistoclakis [6]. (for further preliminaries see [12])

*supported by Hungarian National Foundation for Scientific Research, Grant No. K-61908. Key words: interpolation, near-best approximation, Jacobi weights 2000 MS Classification: 41A05, 41A25 
In this paper we should like to give a de la Vallée Poussin-type interpolatory process in some Jacobi weighted spaces, and deal with the question of approximation of continuous functions. The utility of this method is in the simplicity of the nodes, that is instead of for instance, the roots of orthogonal polynomials, or Fekete or Leja points, we shall interpolate on the nodes $\frac{j \pi}{\nu}, \mathrm{J}=-\nu, \ldots, \nu-1$, and so the computations are very simple. Our starting point was a trigonometric expression of the Christoffel-Darboux kernel of Jacobi-Fourier series (see Szegö 9.3.5 [13]). Summarizing the main-part of this formula from $l+1$ to $2 n$ (the tail-part does not tends to zero with $n$ !), we can construct our interpolatory operator: $M_{n, l}^{(\alpha, \beta)}(f, \vartheta)$. By the notations:

Definition 1 Let $w_{\alpha, \beta}(x)=(1-x)^{\alpha}(1+x)^{\beta}$ be the Jacobi weight function on $(-1,1)(\alpha, \beta>-1)$, and the corresponding weight function on $(-\pi, \pi)$ : $\tilde{w}_{\alpha, \beta}(\varphi)=2^{\alpha+\beta+1} \sin ^{2 \alpha+1} \frac{\varphi}{2} \cos ^{2 \beta+1} \frac{\varphi}{2}$, and for simplicity let us denote by $v_{p, q}(\varphi)=$ $\sin ^{p} \frac{\varphi}{2} \cos ^{q} \frac{\varphi}{2}$.

Furthermore let us define the following classes of continous functions:

\section{Definition 2}

$C_{w_{\alpha, \beta}}[-1,1]=\left\{f: f w_{\alpha, \beta}\right.$ is continuous on $[-1,1], \lim _{x \rightarrow-1} f w_{\alpha, \beta}(x)=0$, if $\beta>0$,

$$
\text { and } \left.\lim _{x \rightarrow 1} f w_{\alpha, \beta}(x)=0, \text { if } \alpha>0\right\}
$$

and

$$
\begin{gathered}
C_{v_{p, q}}[-\pi, \pi]=\left\{f: f v_{p, q} \text { is continuous on }[-\pi, \pi], \lim _{\substack{\varphi \rightarrow-\pi \\
\varphi \rightarrow \pi}}\left(f v_{p, q}\right)(\varphi)=0 \text {, if } q>0\right. \text {, } \\
\text { and } \left.\lim _{\varphi \rightarrow 0}\left(f v_{p, q}\right)(\varphi)=0 \text {, if } p>0\right\}
\end{gathered}
$$

Let us define the interpolatory operators as:

Definition 3 For an $f \in C_{v_{\xi, \eta}}$ (with some $\xi, \eta>-1$ ) let us define the following discrete operators on $[0, \pi)$ for $n \in \mathbb{N}, 0 \leq l<2 n$ and $\nu<n+\frac{l+\beta}{2}+\frac{5}{4}$ :

$$
M_{n, l}^{(\alpha, \beta)}(f, \vartheta)=\frac{2 \pi}{2 n+l+\alpha+\beta+3} \sum_{j=0}^{\nu} f\left(\varphi_{j}\right) \tilde{w}_{\alpha, \beta}\left(\varphi_{j}\right) K_{n}^{l}\left(\varphi_{j}, \vartheta\right)
$$

where

$$
\begin{gathered}
\varphi_{j}=\frac{2 j \pi-2 \gamma}{2 n+l+\alpha+\beta+3}, \quad j=0, \ldots, \nu, \\
\gamma=-\left(\alpha+\frac{1}{2}\right) \frac{\pi}{2}
\end{gathered}
$$

and

$K_{n}^{l}(\varphi, \vartheta)=\frac{k(\varphi) k(\vartheta)}{2^{\alpha+\beta+2}(2 n-l)}\left\{\frac{\sin \left[(2 n+l+\alpha+\beta+3) \frac{\varphi+\vartheta}{2}+2 \gamma\right] \sin (2 n-l) \frac{\varphi+\vartheta}{2}}{\sin ^{2} \frac{\varphi+\vartheta}{2}}\right.$ 


$$
\left.+\frac{\sin (2 n+l+\alpha+\beta+3) \frac{\varphi-\vartheta}{2} \sin (2 n-l) \frac{\varphi-\vartheta}{2}}{\sin ^{2} \frac{\varphi-\vartheta}{2}}\right\}
$$

where

$$
k(\varphi)=\frac{1}{\sqrt{\pi} \sin ^{\alpha+\frac{1}{2}} \frac{\varphi}{2} \cos ^{\beta+\frac{1}{2}} \frac{\varphi}{2}}
$$

At first we have to note that as it can be easily seen that $\forall i, j=0, \ldots, \nu$,

$$
K_{n}^{l}\left(\varphi_{i}, \varphi_{j}\right)=\left\{\begin{array}{l}
0 ; \text { if } i \neq j \\
\frac{k^{2}\left(\varphi_{j}\right)}{2^{\alpha+\beta+2}}(2 n+l+\alpha+\beta+3) ; \text { if } i=j
\end{array}\right.
$$

therefore if $M_{n, l}^{(\alpha, \beta)}(f, \vartheta)$ exists, we get that

$$
M_{n, l}^{(\alpha, \beta)}\left(f, \varphi_{j}\right)=f\left(\varphi_{j}\right), \quad j=0, \ldots, \nu
$$

On the other hand

$$
\begin{gathered}
\tilde{w}_{\alpha, \beta}(\varphi) K_{n}^{l}(\varphi, \vartheta)=\frac{1}{2 \pi} \frac{\sin ^{\alpha+\frac{1}{2}} \frac{\varphi}{2} \cos ^{\beta+\frac{1}{2}} \frac{\varphi}{2}}{\sin ^{\alpha+\frac{1}{2}} \frac{\vartheta}{2} \cos ^{\beta+\frac{1}{2}} \frac{\vartheta}{2}} \frac{1}{2 n-l} \\
\times \sum_{k=l+1}^{2 n}\left\{\frac{\sin \left[\left(k+1+\frac{\alpha+\beta}{2}\right)(\varphi+\vartheta)+2 \gamma\right]}{\sin \frac{\varphi+\vartheta}{2}}+\frac{\sin \left(k+1+\frac{\alpha+\beta}{2}\right)(\varphi-\vartheta)}{\sin \frac{\varphi-\vartheta}{2}}\right\}
\end{gathered}
$$

Denoted by $\frac{\alpha+\beta+1}{2}=M+\delta$, where $M \in \mathbb{Z}, \delta \in[0,1)$, we can expound the expression in the bracket as

$$
\begin{gathered}
\frac{\sin \left[\left(k+1+\frac{\alpha+\beta}{2}\right)(\varphi+\vartheta)+2 \gamma\right]}{\sin \frac{\varphi+\vartheta}{2}}+\frac{\sin \left(k+1+\frac{\alpha+\beta}{2}\right)(\varphi-\vartheta)}{\sin \frac{\varphi-\vartheta}{2}} \\
=2 \sum_{m=0}^{k+M} \cos [(m+\delta)(\varphi+\vartheta)+2 \gamma]+\frac{\sin \left[\left(\delta-\frac{1}{2}\right)(\varphi+\vartheta)+2 \gamma\right]}{\sin \frac{\varphi+\vartheta}{2}} \\
+2 \sum_{m=0}^{k+M} \cos (m+\delta)(\varphi-\vartheta)+\frac{\sin \left(\delta-\frac{1}{2}\right)(\varphi-\vartheta)}{\sin \frac{\varphi-\vartheta}{2}} \\
=4 \sum_{m=0}^{k+M} \cos [(m+\delta)(\varphi)+\gamma] \cos [(m+\delta)(\vartheta)+\gamma] \\
+\frac{\sin \left[\left(\delta-\frac{1}{2}\right)(\varphi+\vartheta)+2 \gamma\right]}{\sin \frac{\varphi+\vartheta}{2}}+\frac{\sin \left(\delta-\frac{1}{2}\right)(\varphi-\vartheta)}{\sin \frac{\varphi-\vartheta}{2}}
\end{gathered}
$$

That is

$$
M_{n, l}^{(\alpha, \beta)}(f, \vartheta)=\frac{2 \pi}{2 n+l+\alpha+\beta+3} \sum_{j=0}^{\nu} f\left(\varphi_{j}\right) \frac{1}{2 \pi} \frac{\sin ^{\alpha+\frac{1}{2}} \frac{\varphi_{j}}{2} \cos ^{\beta+\frac{1}{2}} \frac{\varphi_{j}}{2}}{\sin ^{\alpha+\frac{1}{2}} \frac{\vartheta}{2} \cos ^{\beta+\frac{1}{2}} \frac{\vartheta}{2}}
$$




$$
\begin{gathered}
\times\left\{\left(\frac{4}{2 n-l} \sum_{k=l+1}^{2 n} \sum_{m=0}^{k+M} \cos \left[(m+\delta)\left(\varphi_{j}\right)+\gamma\right] \cos [(m+\delta)(\vartheta)+\gamma]\right)\right. \\
\left.+\left(\frac{\sin \left[\left(\delta-\frac{1}{2}\right)\left(\varphi_{j}+\vartheta\right)+2 \gamma\right]}{\sin \frac{\varphi_{j}+\vartheta}{2}}+\frac{\sin \left(\delta-\frac{1}{2}\right)\left(\varphi_{j}-\vartheta\right)}{\sin \frac{\varphi_{j}-\vartheta}{2}}\right)\right\}
\end{gathered}
$$

In the followings, we want to construct an operator extended on $[-\pi, \pi)$, which is bounded, and for which a reproducing property is valid. Because of this extension, some values of $f$ may appear more than once, so we have to introduce some $\varepsilon_{j}$ constants, to neutralize this occurence, that is we have to use $\varepsilon_{j} f\left(\varphi_{j}\right)$ instead of $f\left(\varphi_{j}\right)$.

To guarantee some reproducing property of $M_{n, l}^{(\alpha, \beta)}(f, \vartheta)$, we have to handle somehow the last two summands. For the purpose of this, we will choose $\alpha+\frac{1}{2}=$ $p$ and $\beta+\frac{1}{2}=q$, where $p$ and $q$ are integers. In this case, since $\delta=\left\{\frac{p+q}{2}\right\}$, thence $\delta=0$ or $\delta=\frac{1}{2}$. Furthermore

$$
\begin{gathered}
\frac{\sin \left[\left(\delta-\frac{1}{2}\right)\left(\varphi_{j}+\vartheta\right)+2 \gamma\right]}{\sin \frac{\varphi_{j}+\vartheta}{2}}+\frac{\sin \left(\delta-\frac{1}{2}\right)\left(\varphi_{j}-\vartheta\right)}{\sin \frac{\varphi_{j}-\vartheta}{2}} \\
=C=\left\{\begin{array}{l}
0, \text { if } \delta=\frac{1}{2} \text { or } p=2 \varrho+1 \\
-2, \text { if } \delta=0 \text { and } p=2 \varrho
\end{array}\right.
\end{gathered}
$$

In the followings we will deal with only these types of parameters. (Here we have to mention that we have another possibility to define an interpolatorytype system of nodes: $\varphi_{j}=\frac{2 j \pi}{2 n-l}$, but it is well-defined on $[-\pi, \pi)$ if and only if $\alpha+\frac{1}{2} \in \mathbb{Z}$, and if $\alpha+\beta \in \mathbb{Z}$, that is we have to choose $p$ and $q$ to be integers again.)

\section{Notations, Result}

With the above notations, in the followings, let $\alpha+\frac{1}{2}=p, \beta+\frac{1}{2}=q$ with $p, q \in \mathbb{Z}$. Now, we can choose

$$
\varphi_{j}=\frac{2 j \pi}{2 n+l+p+q+2}=\frac{j \pi}{\nu}, \quad j=-\nu, \ldots, \nu-1
$$

and $l=n$ or $l=n+1$, according to the pairity of $p+q$, that is $\nu=\frac{2 n+l+p+q}{2} \in \mathbb{N}$. (In the followings $K_{n}^{n}(\varphi, \vartheta)=K_{n}(\varphi, \vartheta)$, and the same for $K_{n}^{n+1}, M_{n, n}^{(p, q)}, M_{n, n+1}^{(p, q)}$. Furthermore let $N=2 n-l$, and $3 N=2 n+l$.) Since $2 \gamma=-p \pi$, we have that

$$
\begin{aligned}
K_{n}(\varphi, \vartheta)= & \frac{k(\varphi) k(\vartheta)}{2^{p+q+1} N}\left\{\frac{(-1)^{p} \sin (3 N+p+q+2) \frac{\varphi+\vartheta}{2} \sin N \frac{\varphi+\vartheta}{2}}{\sin ^{2} \frac{\varphi+\vartheta}{2}}\right. \\
& \left.+\frac{\sin (3 N+p+q+2) \frac{\varphi-\vartheta}{2} \sin N \frac{\varphi-\vartheta}{2}}{\sin ^{2} \frac{\varphi-\vartheta}{2}}\right\}
\end{aligned}
$$


and so

$$
2^{p+q+1} v_{p, q}\left(\varphi_{i}\right) v_{p, q}\left(\varphi_{j}\right) K_{n}\left(\varphi_{i}, \varphi_{j}\right)=\frac{3 N+\alpha+\beta+3}{2 \pi}\left\{\begin{array}{l}
0 ; \text { if } i \neq \pm j \\
1+(-1)^{p} ; \text { if } i=j=0 \\
1+(-1)^{q} \text { if } \varphi_{i}=\varphi_{j}=-\pi \\
1 ; \text { if } \varphi_{i}=\varphi_{j} \neq 0,-\pi \\
1 ; \text { if } i=-j \neq 0
\end{array}\right.
$$

therefore if $M_{n}^{(p, q)}(f, \vartheta)$ exists, then

$$
\begin{gathered}
M_{n}^{(p, q)}(f, 0)=\frac{1+(-1)^{p}}{2} f(0) ; M_{n}^{(p, q)}(f,-\pi)=\frac{1+(-1)^{q}}{2} f(-\pi) ; \\
M_{n}^{(p, q)}\left(f, \varphi_{j}\right)=\frac{f\left(\varphi_{j}\right)+f\left(-\varphi_{j}\right)}{2}, \quad j=\{-\nu+1, \ldots, \nu-1\} \backslash 0
\end{gathered}
$$

\section{Notation:}

(1)With the above notations, we define a trigonometric polynomial $(u(\vartheta))$ such that

$$
\begin{gathered}
u(\vartheta)=u_{p, q}(\vartheta) \\
=\left\{\begin{array}{l}
\sin ^{p} \frac{\vartheta}{2} \cos ^{q+1} \frac{\vartheta}{2}=\sum_{t=0}^{\varrho+\sigma+1} c_{t}(p, q) \cos t \vartheta, \text { if } p=2 \varrho, q=2 \sigma+1 \\
\sin ^{p+1} \frac{\vartheta}{2} \cos ^{q} \frac{\vartheta}{2}=\sum_{t=0}^{\varrho+\sigma+1} c_{t}(p, q) \cos t \vartheta, \text { if } p=2 \varrho+1, q=2 \sigma \\
\sin ^{p} \frac{\vartheta}{2} \cos ^{q} \frac{\vartheta}{2}=\sum_{t=0}^{\varrho+\sigma} c_{t}(p, q) \cos t \vartheta, \text { if } p=2 \varrho, q=2 \sigma \\
\sin ^{p} \frac{\vartheta}{2} \cos ^{q} \frac{\vartheta}{2}=\sum_{t=1}^{\varrho+\sigma+1} c_{t}(p, q) \sin t \vartheta, \text { if } p=2 \varrho+1, q=2 \sigma+1
\end{array}\right.
\end{gathered}
$$

(2) Therefore

$$
\begin{gathered}
M_{n}^{(p, q)}(f, \vartheta) u_{p, q}(\vartheta)=u_{p, q}(\vartheta) \frac{2 \pi}{4 \nu} \sum_{j=-\nu}^{\nu-1} f\left(\varphi_{j}\right) u_{p, q}\left(\varphi_{j}\right) \frac{v_{p, q}\left(\varphi_{j}\right)}{u_{p, q}\left(\varphi_{j}\right)} K_{n}\left(\varphi_{j}, \vartheta\right) \\
=\frac{1}{4 \nu N} \sum_{j=-\nu}^{\nu-1} f\left(\varphi_{j}\right) u_{p, q}\left(\varphi_{j}\right) \frac{v_{p, q}\left(\varphi_{j}\right)}{u_{p, q}\left(\varphi_{j}\right)} \frac{u_{p, q}(\vartheta)}{v_{p, q}(\vartheta)} \\
\times\left\{\frac{(-1)^{p} \sin (2 \nu) \frac{\varphi_{j}+\vartheta}{2} \sin N \frac{\varphi_{j}+\vartheta}{2}}{\sin ^{2} \frac{\varphi_{j}+\vartheta}{2}}+\frac{\sin (2 \nu) \frac{\varphi_{j}-\vartheta}{2} \sin N \frac{\varphi_{j}-\vartheta}{2}}{\sin ^{2} \frac{\varphi_{j}-\vartheta}{2}}\right\}
\end{gathered}
$$

(3) Let $\xi, \eta \in \frac{1}{2} \mathbb{Z}, x \in[-1,1]$, and $x_{j}=\cos \varphi_{j}=\cos \frac{j \pi}{\nu}$.

$$
\begin{gathered}
\left.w_{\xi_{1}, \eta_{1}} M_{n}^{(\xi, \eta)}(f, x)\right)=\frac{1}{N 2 \nu}\left(\frac{1}{2} f\left(x_{0}\right) w_{\xi_{1}, \eta_{1}}\left(x_{0}\right) L_{n}\left(x, x_{0}\right)\right. \\
\left.+\sum_{j=1}^{\nu-1} f\left(x_{j}\right) w_{\xi_{1}, \eta_{1}}\left(x_{j}\right) L_{n}\left(x, x_{j}\right)+\frac{1}{2} f\left(x_{\nu}\right) w_{\xi_{1}, \eta_{1}}\left(x_{\nu}\right) L_{n}\left(x, x_{\nu}\right)\right),
\end{gathered}
$$


where

$$
\begin{gathered}
L_{n}\left(x, x_{j}\right)=\frac{w_{\xi_{1}-\xi, \eta_{1}-\eta}(x)}{w_{\xi_{1}-\xi, \eta_{1}-\eta}\left(x_{j}\right)} \\
\times\left\{\frac{(-1)^{2 \xi} \sin 2 \nu \frac{\arccos x_{j}+\arccos x}{2} \sin N \frac{\arccos x_{j}+\arccos x}{2}}{\sin ^{2} \frac{\arccos x_{j}+\arccos x}{2}}\right. \\
\left.+\frac{\sin 2 \nu \frac{\arccos x_{j}-\arccos x}{2} \sin N \frac{\arccos x_{j}-\arccos x}{2}}{\sin ^{2} \frac{\arccos x_{j}-\arccos x}{2}}\right\}
\end{gathered}
$$

(4) Let us denote by $\|(\cdot)\|$ the infinity norm on the interval in question, either on $[-\pi, \pi]$ or on $[-1,1]$.

\section{Definition 4}

$$
E_{n}^{w}(f)=\min _{\substack{p \in \mathcal{P}_{n} \\ o r \\ p \in \mathcal{T}_{n}}}\|(f-p) w\|
$$

is the error of the unifomly best approximating polynomial/trigonometric polynomial ( $p$ )with degree $n$, with respect to a weight: $w$.

Theorem 1 Let $\xi, \eta \in \frac{1}{2} \mathbb{Z}$, and $\xi, \eta \geq 0$.

$$
f \in C_{w_{\xi_{1}, \eta_{1}}}[-1,1]
$$

where $\left(\xi_{1}, \eta_{1}\right)=\left(\xi, \eta+\frac{1}{2}\right)$, if $2 \xi$ is even, $2 \eta$ is odd; $\left(\xi_{1}, \eta_{1}\right)=\left(\xi+\frac{1}{2}, \eta\right)$, if $2 \xi$ is odd, $2 \eta$ is even, $\left(\xi_{1}, \eta_{1}\right)=(\xi, \eta)$, if $\xi+\eta \in \mathbb{Z}$. Then

$$
w_{\xi_{1}, \eta_{1}}\left(x_{i}\right) M_{n}^{(\xi, \eta)}\left(f, x_{i}\right)=w_{\xi_{1}, \eta_{1}}\left(x_{i}\right) f\left(x_{i}\right), \quad i=0, \ldots, \nu
$$

and

$$
\left\|\left(f(x)-M_{n}^{(\xi, \eta)}(f, x)\right) w_{\xi_{1}, \eta_{1}}\right\|=O\left(E_{n}^{w_{\xi_{1}, \eta_{1}}}(f)\right)
$$

\section{Remark:}

By the same arguments one can prove that $\left\|\left(f(x)-M_{n, l}^{(\xi, \eta)}(f, x)\right) w_{\xi_{1}, \eta_{1}}\right\| \leq$ $c \frac{2 n+l+p+q+2}{2(2 n-l)} E_{l}^{w_{\xi_{1}, \eta_{1}}}(f)$, with $0 \leq l \leq 2 n-1$.

\section{Proof}

For the proof of the theorem, by the substitution $x=\cos \vartheta$ we can work on $[0, \pi)$, and then we can extend the funtion, and the operator as well to $[-\pi, \pi)$. So our first lemma is

Lemma 1 Let $p$ and $q$ be nonnegative integers, and let $f \in C_{u_{p, q}}[-\pi, \pi]$ such that $f$ is even on $(-\pi, 0) \cup(0, \pi)$, then

$$
\left\|\left(f(\vartheta)-M_{n}^{(p, q)}(f, \vartheta)\right) u_{p, q}(\vartheta)\right\|=O\left(E_{n}^{u_{p, q}}(f)\right)
$$




\section{Remark:}

In the followings we will use $l=n$ or $l=n+1$ again, if it is necessary.

$$
\begin{gathered}
M_{n}^{(p, q)}(f, \vartheta) u(\vartheta)=\frac{1}{3 N+p+q+2} \sum_{j=-\nu}^{\nu-1} f\left(\varphi_{j}\right) u\left(\varphi_{j}\right)\left(\frac{C}{2}+\frac{2}{N}\right. \\
\times \sum_{k=l+1}^{2 n} \sum_{m=0}^{k+M}\left\{\begin{array}{l}
\frac{\cos \frac{\vartheta}{2} \cos \left(m+\frac{1}{2}\right)\left(\varphi_{j}\right) \cos \left(m+\frac{1}{2}\right)(\vartheta)}{\cos \frac{\varphi_{j}}{2}}, \text { if } p=2 \varrho, q=2 \sigma+1 \\
\frac{\sin \frac{\vartheta}{2} \sin \left(m+\frac{1}{2}\right)\left(\varphi_{j}\right) \sin \left(m+\frac{1}{2}\right)(\vartheta)}{\sin \frac{\varphi_{j}}{2}}, \text { if } p=2 \varrho+1, q=2 \sigma \\
\cos m \varphi_{j} \cos m \vartheta, \text { if } p=2 \varrho, q=2 \sigma \\
\sin m \varphi_{j} \sin m \vartheta, \text { if } p=2 \varrho+1, q=2 \sigma+1
\end{array}\right)
\end{gathered}
$$

Let us call the above cases as case (a),(b),(c) and (d) respectively. The previous computations show that

Lemma $2 M_{n}^{(p, q)}(f, \vartheta) u(\vartheta)$ in case (a) and $(b)$ is a cosine-polynomial with degree $2 n+M+1$; in case (c) is also a cosine polynomial, but with degree $2 n+M$; and in case (d) is a sine-polynomial with degree $2 n+M$.

Now we can turn to the proof of the reproducing property:

Lemma 3 Let $C_{L}(\vartheta)$ be a cosine-polynomial with degree $L$ on $[-\pi, \pi)$. If $L \leq n$, then

$$
C_{L}(\vartheta) u(\vartheta)=M_{n}^{(p, q)}\left(C_{L}, \vartheta\right) u(\vartheta), \quad \vartheta \in[-\pi, \pi) .
$$

\section{Remark:}

Naturally in the above expression $u(\vartheta)$ is necessary only that points, in which it is zero.

\section{Proof:}

At first we will deal with case $(a)$. Now

$$
\begin{gathered}
M_{n}^{(p, q)}(f, \vartheta) u(\vartheta)=\frac{1}{(3 N+p+q+2) N} \sum_{j=-\nu}^{\nu-1} f\left(\varphi_{j}\right) u\left(\varphi_{j}\right) \\
\times \sum_{k=l+1}^{2 n} \sum_{m=0}^{k+M} a_{m, j}(\cos m \vartheta+\cos (m+1) \vartheta),
\end{gathered}
$$

where

$$
a_{m, j}=\frac{\cos \left(m+\frac{1}{2}\right) \varphi_{j}}{\cos \frac{\varphi_{j}}{2}}, \quad a_{0, j}=a_{0}=1
$$

That is

$$
\begin{aligned}
M_{n}^{(p, q)} & (f, \vartheta) u(\vartheta)=\frac{1}{3 N+p+q+2} \sum_{j=-\nu}^{\nu-1} f\left(\varphi_{j}\right) u\left(\varphi_{j}\right) \\
& \times\left\{a_{0}+\sum_{m=1}^{l+M+1}\left(a_{m-1, j}+a_{m, j}\right) \cos m \vartheta\right.
\end{aligned}
$$




$$
\begin{gathered}
+\sum_{m=l+1+M+1}^{2 n+M} \frac{2 n+M+1-m}{N}\left(a_{m-1, j}+a_{m, j}\right) \cos m \vartheta \\
\left.+\sum_{m=l+1+M+1}^{2 n+M+1} \frac{a_{m-1, j}}{N} \cos m \vartheta\right\}
\end{gathered}
$$

Let $f(\vartheta)=C_{L}(\vartheta)$ is a cosine polynomial with degree $L$ now!

Then taking into consideration that

(i) in case (a) $C_{L}(\vartheta) u(\vartheta)=\sum_{\mu=0}^{L+M+1} d_{\mu} \cos \mu \vartheta$ is also a cosine polynomial,

(ii) $\beta>-1, C_{l}\left(\varphi_{-\nu}\right) u\left(\varphi_{-\nu}\right)=0=\sum_{\mu=0}^{L+M+1}(-1)^{\mu} d_{\mu}$,

(iii) $a_{m-1, j}+a_{m, j}=2 \cos m \varphi_{j}$,

(iv) $a_{m, j}=(-1)^{m}\left(2 \sum_{\varrho=1}^{m}(-1)^{\varrho} \cos \varrho \varphi_{j}+1\right)$

we get that

$$
\begin{gathered}
M_{n}^{(p, q)}\left(C_{L}, \vartheta\right) u(\vartheta)=\frac{1}{2 \nu} \sum_{j=-\nu}^{\nu-1}\left(\sum_{\mu=0}^{L+M+1} d_{\mu} \cos \mu \varphi_{j}\right. \\
\times\left\{a_{0}+2 \sum_{m=1}^{l+M+1} \cos m \varphi_{j} \cos m \vartheta+2 \sum_{m=l+1+M+1}^{2 n+M} \frac{2 n+M+1-m}{N} \cos m \varphi_{j} \cos m \vartheta\right. \\
\left.\left.+\frac{1}{N} \sum_{m=l+1+M+1}^{2 n+M+1}(-1)^{m-1} \cos m \vartheta\left(2 \sum_{\varrho=1}^{m-1}(-1)^{\varrho} \cos \varrho \varphi_{j}+1\right)\right\}\right)
\end{gathered}
$$

Changing the order of summations, we can write

$$
\begin{gathered}
M_{n}^{(p, q)}\left(C_{L}, \vartheta\right) u(\vartheta)=\frac{1}{2 \nu} \sum_{\mu=0}^{L+M+1} d_{\mu} \\
\times\left\{\sum_{j=-\nu}^{\nu-1} \cos \mu \varphi_{j}+2 \sum_{m=1}^{l+M+1} \cos m \vartheta \sum_{j=-\nu}^{\nu-1} \cos \mu \varphi_{j} \cos m \varphi_{j}\right. \\
+\frac{2}{N} \sum_{m=l+1+M+1}^{2 n+M}(2 n+M+1-m) \cos m \vartheta \sum_{j=-\nu}^{\nu-1} \cos \mu \varphi_{j} \cos m \varphi_{j} \\
\left.+\frac{1}{N} \sum_{m=l+1+M+1}^{2 n+M+1}(-1)^{m-1} \cos m \vartheta\left(2 \sum_{\varrho=1}^{m-1}(-1)^{\varrho} \sum_{j=-\nu}^{\nu-1} \cos \mu \varphi_{j} \cos \varrho \varphi_{j}+\sum_{j=-\nu}^{\nu-1} \cos \mu \varphi_{j}\right)\right\}
\end{gathered}
$$

Since

$$
\sum_{j=-\nu}^{\nu-1} \cos m \varphi_{j} \cos \mu \varphi_{j}=\left\{\begin{array}{l}
0, \text { if } m \neq \mu \\
2 \nu, \text { if } m=\mu=0 \\
\nu, \text { if } m=\mu \neq 0
\end{array},\right.
$$

and according to (ii), for an $L \leq l$ 


$$
\begin{gathered}
M_{n}^{(p, q)}\left(C_{L}, \vartheta\right) u(\vartheta)=\frac{1}{2 \nu}\left\{2 \nu d_{0}+2 \sum_{m=1}^{L+M+1} d_{\mu} \nu \cos \mu \vartheta+0\right. \\
\left.+\frac{1}{N} \sum_{m=L+1+M+1}^{2 n+M+1}(-1)^{m-1} \cos m \vartheta\left(2 \nu \sum_{\mu=0}^{L+M+1}(-1)^{\mu} d_{\mu}\right)\right\}=C_{L}(\vartheta) u(\vartheta)
\end{gathered}
$$

In case (b)

$$
M_{n}^{(p, q)}(f, \vartheta) u(\vartheta)=\frac{1}{2 \nu N} \sum_{j=-\nu}^{\nu-1} f\left(\varphi_{j}\right) u\left(\varphi_{j}\right) \sum_{k=l+1}^{2 n} \sum_{m=0}^{k+M} b_{m, j}(\cos m \vartheta+\cos (m+1) \vartheta),
$$

where

$$
b_{m, j}=\frac{\sin \left(m+\frac{1}{2}\right) \varphi_{j}}{\sin \frac{\varphi_{j}}{2}}, \quad b_{0, j}=b_{0}=1
$$

So because

$$
b_{m, j}-b_{m-1, j}=2 \cos m \varphi_{j}
$$

$$
M_{n}^{(p, q)}(f, \vartheta) u(\vartheta)=\frac{1}{2 \nu} \sum_{j=-\nu}^{\nu-1} f\left(\varphi_{j}\right) u\left(\varphi_{j}\right)\left\{b_{0}+2 \sum_{m=1}^{l+M+1} \cos m \varphi_{j} \cos m \vartheta\right.
$$

$$
\left.+2 \sum_{m=l+1+M+1}^{2 n+M} \frac{2 n+M+1-m}{N} \cos m \varphi_{j} \cos m \vartheta-\sum_{m=l+1+M+1}^{2 n+M+1} \frac{b_{m-1, j}}{N} \cos m \vartheta\right\}
$$

If $f(\vartheta)=C_{L}(\vartheta)$ is a cosine polynomial with degree $L$, then

(i) in case (b) $C_{L}(\vartheta) u(\vartheta)=\sum_{\mu=0}^{L+M+1} d_{\mu} \cos \mu \vartheta$ is also a cosine polynomial,

(ii) $\alpha>-1, C_{l}\left(\varphi_{0}\right) u\left(\varphi_{0}\right)=0=\sum_{\mu=0}^{L+M+1} d_{\mu}$,

(iii) $b_{m, j}=1+2 \sum_{\varrho=1}^{m} \cos \varrho \varphi_{j}$

And

$$
\begin{aligned}
& M_{n}^{(p, q)}\left(C_{L}, \vartheta\right) u(\vartheta)=\frac{1}{2 \nu}\left\{2 \nu d_{0}+2 \sum_{m=1}^{L+M+1} d_{\mu} \nu \cos \mu \vartheta+0\right. \\
& \left.+\frac{1}{N} \sum_{m=L+1+M+1}^{2 n+M+1} \cos m \vartheta\left(2 \nu \sum_{\mu=0}^{L+M+1} d_{\mu}\right)\right\}=C_{L}(\vartheta) u(\vartheta)
\end{aligned}
$$

In case (c)

$$
M_{n}^{(p, q)}(f, \vartheta) u(\vartheta)=\frac{1}{2 \nu} \sum_{j=-\nu}^{\nu-1} f\left(\varphi_{j}\right) u\left(\varphi_{j}\right)\left\{-1+\frac{2}{N} \sum_{k=l+1}^{2 n} \sum_{m=0}^{k+M} \cos m \varphi_{j} \cos m \vartheta\right\}
$$




$$
\begin{gathered}
=\frac{1}{2 \nu} \sum_{j=-\nu}^{\nu-1} f\left(\varphi_{j}\right) u\left(\varphi_{j}\right) \\
\left\{-1+2 \sum_{m=0}^{l+M+1} \cos m \varphi_{j} \cos m \vartheta+2 \sum_{m=l+1+M+1}^{2 n+M} \frac{2 n+M+1-m}{N} \cos m \varphi_{j} \cos m \vartheta\right\}
\end{gathered}
$$

That is if $f(\vartheta)=C_{L}(\vartheta)$ is a cosine polynomial with degree $L$, then $C_{L}(\vartheta) u(\vartheta)=$ $\sum_{\mu=0}^{L+M} d_{\mu} \cos \mu \vartheta$ is also a cosine polynomial, and for $L \leq n M_{n}^{(p, q)}\left(C_{L}, \vartheta\right) u(\vartheta)=$ $C_{L}(\vartheta) u(\vartheta)$, as in the previous case.

Similarly, in case (d) if $f(\vartheta)=C_{L}(\vartheta)$ is a cosine polynomial with degree $L \leq n$, then $C_{L}(\vartheta) u(\vartheta)=\sum_{\mu=1}^{L+M} d_{\mu} \sin \mu \vartheta$ is a sine polynomial, and using the orthogonality of $\sin \varphi_{j}$-S on $[-\pi, \pi]$

$$
\begin{gathered}
M_{n}^{(p, q)}\left(C_{L}, \vartheta\right) u(\vartheta)=\frac{1}{2 \nu} \sum_{j=-\nu}^{\nu-1} \sum_{\mu=1}^{L+M} d_{\mu} \sin \mu \varphi_{j} \\
\times\left\{\frac{2}{N} \sum_{k=l+1}^{2 n} \sum_{m=1}^{k+M} \sin m \varphi_{j} \sin m \vartheta\right\}=C_{L}(\vartheta) u(\vartheta) .
\end{gathered}
$$

Lemma 4 With the previous notations

$$
\begin{gathered}
\Lambda_{n}=\left\|u_{p, q}(\vartheta) \lambda_{n}^{(p, q)}(\vartheta)\right\| \\
=\left\|u_{p, q}(\vartheta) \frac{2 \pi}{2(3 N+p+q+2)} \sum_{j=-\nu}^{\nu-1}\left|\frac{v_{p, q}\left(\varphi_{j}\right)}{u_{p, q}\left(\varphi_{j}\right)} K_{n}\left(\varphi_{j}, \vartheta\right)\right|\right\|=O(1)
\end{gathered}
$$

\section{Proof:}

case (a):

$$
\begin{gathered}
u_{p, q}(\vartheta) \lambda_{n}^{(p, q)}(\vartheta)=\frac{1}{2 \nu N} \\
\times \sum_{j=-\nu}^{\nu-1}\left|\frac{\cos \frac{\vartheta}{2}}{\cos \frac{\varphi_{j}}{2}}\left\{\frac{\sin 2 \nu \frac{\varphi_{j}+\vartheta}{2} \sin N \frac{\varphi_{j}+\vartheta}{2}}{\sin ^{2} \frac{\varphi_{j}+\vartheta}{2}}+\frac{\sin 2 \nu \frac{\varphi_{j}-\vartheta}{2} \sin N \frac{\varphi_{j}-\vartheta}{2}}{\sin ^{2} \frac{\varphi_{j}-\vartheta}{2}}\right\}\right| \\
=\frac{1}{2 \nu} \mid 1+2 \sum_{m=1}^{l+M+1}(-1)^{m} \cos m \vartheta+2 \sum_{m=l+1+M+1}^{2 n+M} \frac{2 n+M+1-m}{N}(-1)^{m} \cos m \vartheta \\
+\sum_{m=l+1+M+1}^{2 n+M+1} \frac{2 m-1}{N}(-1)^{m-1} \cos m \vartheta \mid+\frac{1}{2 \nu N} \\
\times \sum_{j=-\nu+1}^{\nu-1}\left|\frac{\cos \frac{\vartheta}{2}}{\cos \frac{\varphi_{j}}{2}}\left\{\frac{\sin 2 \nu \frac{\varphi_{j}+\vartheta}{2} \sin N \frac{\varphi_{j}+\vartheta}{2}}{\sin ^{2} \frac{\varphi_{j}+\vartheta}{2}}+\frac{\sin 2 \nu \frac{\varphi_{j}-\vartheta}{2} \sin N \frac{\varphi_{j}-\vartheta}{2}}{\sin ^{2} \frac{\varphi_{j}-\vartheta}{2}}\right\}\right|
\end{gathered}
$$




$$
=A+B=A+\frac{1}{2 \nu N} \sum_{j=-\nu+1}^{\nu-1} B_{\nu}(\vartheta)
$$

It is clear, that $A=O(1)$. It is enough to estimate $B$ on $[0, \pi)$, so let $0 \leq \varphi_{k-1} \leq \vartheta<\varphi_{k}$ !

$$
\begin{gathered}
B \leq \frac{1}{2 \nu N}\left(B_{k-1}(\vartheta)+B_{k}(\vartheta)+B_{k+1}(\vartheta)+B-k-1(\vartheta)+B_{-k}(\vartheta)+B_{-k+1}(\vartheta)\right) \\
+\frac{1}{2 \nu N} \sum_{\substack{-\nu+1 \leq j \leq \nu-1 \\
j \neq-k-1,-k,-k+1, k-1, k, k+1}} B_{\nu}(\vartheta)=B_{*}(\vartheta)+B_{* *}(\vartheta)
\end{gathered}
$$

Since $B_{i}(\vartheta) \leq 4 \nu N,(i=k-1, k, k+1,-k-1,-k,-k+1)$ thence $B_{*}(\vartheta)=$ $O(1)$.

$$
\begin{gathered}
B_{* *}(\vartheta) \leq c \frac{1}{2 \nu N}\left\{\sum_{j=-\nu+1}^{-k-2}\left|\frac{\cos \frac{\varphi_{k-1}}{\varphi_{j}}}{\cos \frac{\varphi_{j}}{2}}\right|\left(\frac{1}{\sin ^{2} \frac{\varphi_{j}+\varphi_{k-1}}{2}}+\frac{1}{\sin ^{2} \frac{\varphi_{j}-\varphi_{k-1}}{2}}\right)\right. \\
\left.+\sum_{j=-k+2}^{k-2}(\cdot)+\sum_{k+2}^{\nu-1}(\cdot)\right\} \leq c \frac{\nu^{2}}{\nu N}\left\{\sum_{j=-\nu+1}^{-k-2} \frac{\nu-k}{\nu-|j|}\right. \\
\times\left(\max \left\{\frac{1}{(j+k)^{2}}, \frac{1}{(2 \nu-|j+k|)^{2}}\right\}+\max \left\{\frac{1}{(j-k)^{2}}, \frac{1}{(2 \nu-|j-k|)^{2}}\right\}\right) \\
\left.+\sum_{j=-k+2}^{k-2}(\cdot)+\sum_{k+2}^{\nu}(\cdot)\right\}=\Sigma_{11}+\Sigma_{12}+\Sigma_{21}+\Sigma_{22}+\Sigma_{31}+\Sigma_{32} \\
\Sigma_{11}=c \frac{\nu}{N} \sum_{j=-\nu+1}^{-k-2} \frac{\nu-k}{\nu-|j|} \max \left\{\frac{1}{(j+k)^{2}}, \frac{1}{(2 \nu-|j+k|)^{2}}\right\} \\
=c \frac{\nu}{N}\left\{\sum_{j=k+2}^{\nu-1} \frac{\nu-k}{(\nu-j)(j-k)^{2}}\right\}=O(1) \\
\Sigma_{12}=c \frac{\nu}{N} \sum_{j=-\nu+1}^{-k-2} \frac{\nu-k}{\nu-|j|} \max \left\{\frac{1}{(j-k)^{2}}, \frac{1}{(2 \nu-|j-k|)^{2}}\right\} \\
\left.\Sigma_{21}=c \frac{\nu}{N} \sum_{j=-k+2}^{\nu} \frac{\nu-k}{\nu-k} \frac{\nu-k}{\nu-j} \frac{1}{(j+k)^{2}}+\sum_{j=k+2}^{\nu-k+1} \frac{\nu-k}{\nu-j} \frac{1}{(2 \nu-j-k)^{2}}\right)=O(1) \\
\sum_{j=k}\left\{\frac{1}{(j+k)^{2}}, \frac{1}{(2 \nu-|j+k|)^{2}}\right\}=O(1)
\end{gathered}
$$




$$
\Sigma_{22}=c \frac{\nu}{N} \sum_{j=-k+2}^{k-2} c \frac{1}{(k-j)^{2}}=O(1)
$$

$\Sigma_{3}$ is similar to $\Sigma_{1}$ :

$$
\begin{gathered}
\Sigma_{31}=c \frac{\nu}{N}\left(\sum_{j=k+2}^{\nu-k} \frac{\nu-k}{\nu-j} \frac{1}{(j+k)^{2}}\right. \\
\left.+\sum_{j=\max \{k+2, \nu-k\}+1}^{\nu-1} \frac{\nu-k}{\nu-j} \frac{1}{(2 \nu-j-k)^{2}}\right)=O(1)
\end{gathered}
$$

and

$$
\Sigma_{32}=c \frac{\nu}{N} \sum j=k+2 \nu-1 \frac{\nu-k}{\nu-j} \frac{1}{(j-k)^{2}}=O(1)
$$

Case (b) is very similar to case (a); the only difference is that we have to separate the $\nu$ - 0 -term, so we omit the details.

In cases (c) and (d) $u_{p, q}=v_{p, q}$ so we can follow the above computations without an extra factor.

Proof: of Lemma 1

For an $f$ in Lemma 1, one can give a best approximating polynomial with degree $l$ with respect to $u_{p, q}$, which is a cosine-polynomial, on a standard way. So by Lebesgue's inequality, and according to lemmas 2,3:

$$
\left\|\left(f-M_{n}^{(p, q)}(f)\right) u_{p, q}\right\| \leq\left(1+\Lambda_{n}\right) E_{n}^{u_{p, q}}(f)
$$

and together with Lemma 4, it gives Lemma 1.

Proof: of Theorem 1

Let $f$ be as in Theorem 1 . Then by the replacement $x=\cos \vartheta$, denoting by $f(\vartheta):=f(\cos \vartheta)$ again, an $f \in C_{u_{p, q}}[0, \pi]$ is defined, where $p=2 \xi, q=2 \eta$. Now let us extend this function to $(-\pi, 0) \cup(0 \pi)$, so (denoting also by $f$ ) we have an even function in $C_{u_{p, q}}[-\pi, \pi]$ As in previously, we can define the best approximating polinomial to $f$ with degree $n$, with respect to $w_{\xi_{1}, \eta_{1}}$ on $[-1,1]$. That is $\left\|\left(f(x)-p_{n}(x)\right) w_{\xi_{1}, \eta_{1}}(x)\right\|=E_{n}^{w_{\xi_{1}, \eta_{1}}}(f)$. Substituting $x=\cos \vartheta$, and denoting by $C_{n}(\vartheta)=p_{n}(\cos \vartheta),\left\|\left(f(\vartheta)-C_{n}(\vartheta)\right) u_{p, q}(\vartheta)\right\|=E_{n}^{w_{\xi_{1}}, \eta_{1}}(f(x))=$ $E_{n}^{u_{p, q}}(f(\vartheta))$. Applying Lemma 1, we get that

$$
\begin{gathered}
\left\|\left(f(\vartheta)-M_{n}^{(p, q)}(f, \vartheta)\right) u_{p, q}(\vartheta)\right\|=O(1) E_{n}^{u_{p, q}(f)} \\
\left.M_{n}(f, \vartheta)^{(p, q)}\right) u_{p, q}(\vartheta)=\frac{1}{4 \nu N}\left(\sum_{j=-\nu}^{-1} f\left(\varphi_{j}\right) u_{p, q}\left(\varphi_{j}\right) \frac{v_{p, q}\left(\varphi_{j}\right)}{u_{p, q}\left(\varphi_{j}\right)} \frac{u_{p, q}(\vartheta)}{v_{p, q}(\vartheta)}\right. \\
\times\left\{\frac{(-1)^{p} \sin (2 \nu) \frac{\varphi_{j}+\vartheta}{2} \sin N \frac{\varphi_{j}+\vartheta}{2}}{\sin ^{2} \frac{\varphi_{j}+\vartheta}{2}}+\frac{\sin (2 \nu) \frac{\varphi_{j}-\vartheta}{2} \sin N \frac{\varphi_{j}-\vartheta}{2}}{\sin ^{2} \frac{\varphi_{j}-\vartheta}{2}}\right\}
\end{gathered}
$$




$$
\begin{gathered}
\left.+\sum_{j=0}^{\nu-1}(\cdot)\right)=\frac{1}{4 \nu N}(I+I I) \\
I=\sum_{j=-\nu}^{-1} f\left(\varphi_{-j}\right) \delta u_{p, q}\left(\varphi_{-j}\right) \frac{\varepsilon v_{p, q}\left(\varphi_{-j}\right)}{u_{p, q}\left(\varphi_{-j}\right)} \frac{u_{p, q}(\vartheta)}{v_{p, q}(\vartheta)} \\
\times\left\{\frac{(-1)^{p} \sin (2 \nu) \frac{-\varphi_{-j}+\vartheta}{2} \sin N \frac{-\varphi_{-j}+\vartheta}{2}}{\sin ^{2} \frac{-\varphi_{-j}+\vartheta}{2}}+\frac{\sin (2 \nu) \frac{-\varphi_{-j}-\vartheta}{2} \sin N \frac{-\varphi_{-j}-\vartheta}{2}}{\sin ^{2} \frac{-\varphi_{-j}-\vartheta}{2}}\right\},
\end{gathered}
$$

where $\varepsilon=-1$, if $p$ is odd and $q$ is even, and $\varepsilon=1$ otherwise; $\delta=-1$ if $p$ and $q$ are odd, and $\delta=1$ otherwise, that is $\varepsilon \delta=(-1)^{p}$. So

$$
\begin{gathered}
I=\sum_{j=1}^{\nu} f\left(\varphi_{j}\right) u_{p, q}\left(\varphi_{j}\right) \frac{v_{p, q}\left(\varphi_{j}\right)}{u_{p, q}\left(\varphi_{j}\right)} \frac{u_{p, q}(\vartheta)}{v_{p, q}(\vartheta)} \\
\times\left\{\frac{(-1)^{p} \sin (2 \nu) \frac{\varphi_{j}+\vartheta}{2} \sin N \frac{\varphi_{j}+\vartheta}{2}}{\sin ^{2} \frac{\varphi_{j}+\vartheta}{2}}+\frac{\sin (2 \nu) \frac{\varphi_{j}-\vartheta}{2} \sin N \frac{\varphi_{j}-\vartheta}{2}}{\sin ^{2} \frac{\varphi_{j}-\vartheta}{2}}\right\},
\end{gathered}
$$

and

$$
\begin{gathered}
\left.M_{n}(f, \vartheta)^{(p, q)}\right) u_{p, q}(\vartheta)=\frac{1}{2 \nu N} \\
\times\left(\frac{1}{2} f\left(\varphi_{0}\right) u_{p, q}\left(\varphi_{0}\right) L_{n}\left(\vartheta, \varphi_{0}\right)\right. \\
\left.+\sum_{j=1}^{\nu-1} f\left(\varphi_{j}\right) u_{p, q}\left(\varphi_{j}\right) L_{n}\left(\vartheta, \varphi_{j}\right)+\frac{1}{2} f\left(\varphi_{\nu}\right) u_{p, q}\left(x_{\nu}\right) L_{n}\left(\vartheta, \varphi_{\nu}\right)\right)
\end{gathered}
$$

Substituting $\cos \vartheta=x, \cos \varphi_{j}=x_{j}$ in (35), and taking into consideration (36) the theorem is proved.

\section{Acknowledgement}

The author is thankful to Professor József Szabados for many useful discussions.

\section{References}

[1] R. Bojanic and O. Shisha, Approximation of continuous, periodic functions by discrete positive linear operators, J. of Approximation Theory, 11 (1974) 231-235.

[2] G. Freud, Interpolációs polinomokkal való approximációról, Mat. lapok 18 (1967) 61-64. (in Hungarian)

[3] G. Freud and A. Sharma, Some good sequences of interpolatory polynomials, Canad. J. Math. 26 (1974), 233-246; Addendum, ibid., 29 (1977), 1163-1166. 
[4] O. Kis and J. Szabados, On some de la Vallée Poussin type discrete linear operators, Acta Math. Hung. 47 (1-2) (1986) 239-260.

[5] H. P. Mashele, Mhaskar-Prestin operators for Freud weights, East J. on Approx. 8 (2002) 501-510.

[6] G. Mastroianni and W. Themistoclakis, De la Valee Poussin means and Jackson's theorem, Acta Sci. Math. (Szeged) 74 (2008) 147-170.

[7] H. N. Mhaskar, Polynomial operators and local smoothness classes on the unit interval, J. of Approximation Theory, 131 (2) (2004) 243-267.

[8] H. N. Mhaskar and J. Prestin, Polynomial operators for spectral approximation of piecewise analytic functions, Appl. and Comp. Harmonic Analysis 26 (1) (2009) 121-142.

[9] H. N. Mhaskar and J. Prestin, Bounded quasi-interpolatory polynomial operators, J. of Approximation Theory, 96 (1) (1999) 67-85.

[10] N. X. Ky, On approximation of functions by polynomials with weight, Acta Math. Hung. 59 (1992) 49-58.

[11] J. Szabados, On an interpolatory analogon of the de la Vallée Poussin means, Studia Sci. Math. Hungarica 9 (1974) 187-190.

[12] J. Szabados, Discrete linear interpolatory operators, Surveys in Approx. Theory 2 (2006) 53-60.

[13] G. Szegő, Orthogonal polynomials, AMS Colloquium Publications XXIII Amer. Math. Soc., New York, (1959) 